ORIGINAL ARTICLE

\title{
Body movements on the men's competition mushroom: a three dimensional analysis of circular swings
}

\author{
G Grassi, M Turci, Y F Shirai, N Lovecchio, C Sforza, V F Ferrario
}

Br J Sports Med 2005;39:489-492. doi: 10.1136/bjsm.2003.010256

See end of article for authors' affiliations ......................

Correspondence to: Professor Sforza, Department of Human Morphology, University of Milan, Via Mangiagalli 31, Milano 20133, Italy; chiarella.sforza@unimi.it

Accepted 6 January 2004
Objectives: To develop a method for the three dimensional analysis of body movements and body positions during the performance of circular swings on the competition mushroom, an apparatus used by young gymnasts for pommel horse training.

Methods: Five experienced male gymnasts, all of national level, performed three series of 10 circular swings on the competition mushroom. An optoelectronic instrument was used for the detection of the three dimensional movement of 13 body landmarks. From landmark trajectories, several technical measurements were obtained: diameters of ideal circles of ankles, hips, shoulders; deviation of the ankle diameters from circularity and from the horizontal plane; angle between the shoulder, hip, and ankle. The values were used for a quantitative assessment of performance of the five gymnasts.

Results: During the exercise, each ankle should follow a nearly horizontal circular path (deviation from circularity ranged from $3.6 \%$ to $6 \%$, deviation from horizontality was $9.4-19.7 \%$ ), there should be an angle of about $180^{\circ}$ at the hips (actual values 146-153 ), and the shoulders should move as little as possible, and only in the lateral plane, without major anteroposterior movements (shoulder movement was $27-31 \%$ of ankle movement, hip movement was 16-20\%).

Conclusions: The method could help coaches and gymnasts to determine which parts of the body are not repeating a selected movement with sufficient accuracy and to quantify improvements made after a specific training programme.
$\mathrm{D}$ uring the execution of artistic gymnastics exercises the judges must evaluate each performance "objectively, accurately, consistently, ethically, fairly, and quickly". ${ }^{1}$ To this end, detailed codes of points are prepared by the international federation and constantly reviewed according to the characteristics of an evolving discipline. ${ }^{2}$ The primary purpose of each code of points is "to provide an objective means"1 of evaluation. In the code, each exercise is given a particular value, mainly depending on its difficulty.

During competitions, the actual execution of a gymnast is evaluated considering the errors related to technique and body position. ${ }^{1}$ The different body positions in space, as well as the reciprocal arrangement of the various parts of the body, determine the aesthetic aspects of the exercise. Indeed, all parts of the body must harmonise to allow a successful and appreciated execution. ${ }^{2-4}$ The same criteria are obviously used by coaches during daily training.

Current technology allows the three dimensional detection and recording of fast and complex movements and the quantitative analysis of the spatial and temporal patterns of motion of body landmarks..$^{4-7}$ In investigations performed on gymnastics, data are mostly used as input values for simulation models. ${ }^{67}$ These data could also be used to provide measurements similar to those qualitatively assessed by judges and coaches.

The aim of this study was to develop a method for the three dimensional analysis of body movements and body positions during the performance of circular swings on the competition mushroom, an apparatus used by young gymnasts for pommel horse training. Preliminary data on five gymnasts are reported.

\section{METHODS}

Participants

Five experienced male gymnasts, all in good health, volunteered for the study (table 1). They were all of national level, and had 7-14 years of specific training in gymnastics. After all the experimental procedures and possible discomforts and risks of the study had been fully described, written consent was obtained from each participant. The protocol used was approved by the local ethics committee.

Body weight was measured to the nearest $0.5 \mathrm{~kg}$ on a beam balance scale, and stature was measured to the nearest $1 \mathrm{~cm}$ with a stadiometer. Before data collection, body composition was estimated by bioelectric impedance analysis performed with a BIA 101/SC instrument (Akern srl, Firenze, Italy) using the standardised protocol described by the manufacturer. Percentage body fat-free mass estimates were obtained using software provided by the manufacturer. ${ }^{8}$

\section{Movement studied}

Spherical retro-reflective markers (diameter $1 \mathrm{~cm}$ ), corresponding to 13 anatomical landmarks, were positioned on the body of each gymnast (fig 1). In the same session and after a warming up period, each participant performed three series of 10 circular swings on the competition mushroom, with a five minute rest between each series. All the participants were told to perform their best.

During the exercise, each ankle should follow a nearly horizontal circular path, there should be an angle of about $180^{\circ}$ at the hips, and the shoulders should move as little as possible, and only in the lateral plane, without major anteroposterior movements.

\section{Film acquisition}

An optoelectronic computerised instrument (Elite System, BTS, Milan, Italy) was used for data acquisition. It allowed the automatic analysis of the movement from the three dimensional coordinates of different body landmarks which were detected by eight infrared sensitive, charge coupled device cameras working at $100 \mathrm{~Hz} .{ }^{9}{ }^{10}$ The eight cameras were positioned at variable heights from the floor and at various 
Table 1 Basic details of the five gymnasts analysed

\begin{tabular}{llllll}
\hline Gymnast & $\begin{array}{l}\text { Age } \\
\text { (years) }\end{array}$ & $\begin{array}{l}\text { Height } \\
(\mathbf{c m})\end{array}$ & $\begin{array}{l}\text { Weight } \\
\mathbf{( k g )}\end{array}$ & $\begin{array}{l}\text { BMI } \\
\left(\mathbf{k g} / \mathbf{m}^{2}\right)\end{array}$ & $\begin{array}{l}\% \text { FFM } \\
(\%)\end{array}$ \\
\hline M1 & 21 & 162 & 74 & 28.20 & 86.5 \\
M2 & 21 & 177 & 63 & 20.11 & 81.6 \\
M3 & 20 & 160 & 62 & 24.22 & 76.8 \\
M4 & 18 & 165 & 62 & 22.77 & 84.0 \\
M5 & 21 & 176 & 75 & 24.21 & 88.0 \\
Mean & 20.2 & 168 & 67.2 & 23.90 & 83.4 \\
SD & 1.3 & 8.0 & 6.7 & 2.93 & 4.4 \\
\hline
\end{tabular}

BMI, Body mass index, ratio of body weight to squared standing height; \%FFM, percentage fat-free mass, estimated from bioelectric impedance analysis.

angles of a working volume of $380 \times 380 \times 280 \mathrm{~cm}$ to film each participant from different corners and points of view.

Before each acquisition session, a metric calibration and correction of optical and electronic distortions was performed. During the execution of the movement, special software recognised the coordinates of the centre of gravity of each marker for any TV camera. Subsequently, all the coordinates were converted into real metric data, and a set of $\mathrm{x}, \mathrm{y}, \mathrm{z}$ coordinates for each landmark in each frame which constituted the movement was obtained..$^{9}$

\section{Data analysis}

Data were analysed separately for each gymnast and each series of 10 circular swings.

For each swing, the three dimensional coordinates of the right and left lateral malleoli (ankles), anterior superior iliac spines (hips), and acromia (shoulders) were used to compute the ideal circles projected on the horizontal plane as follows. From all the time frames of the swing (mean 1393, range 1290-1490), the centre of gravity of the trajectory of the

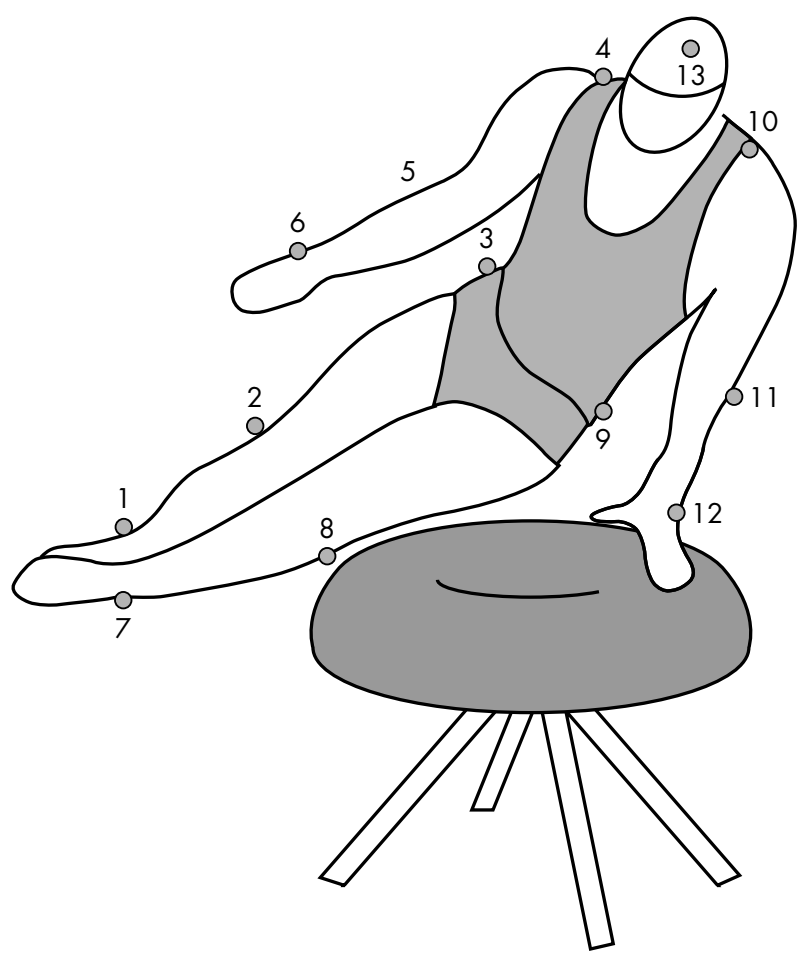

Figure 1 Anatomical landmarks analysed as the gymnast performs a circular swing on the competition mushroom: 1, right lateral malleolus (ankle); 2, right fibular head (knee); 3, anterior superior iliac spine (hip); 4, right acromion (shoulder); 5, right olecranon (elbow); 6, right styloid process of the ulna (wrist); 7, left ankle; 8 , left knee; 9, left hip; 10, left shoulder; 11, left elbow; 12, left wrist; 13, vertex (head). landmark was calculated, the trajectory was projected on the horizontal plane passing through its centre of gravity, and the radius between the position of the landmark in each time frame and the centre of gravity was computed.

The diameters $(\mathrm{mm})$ of the left and right ideal circles (ankles, hips, shoulders) were obtained. For each series, descriptive statistics of the 10 diameters were calculated. From the ankle diameters, the coefficient of variation (percentage ratio of standard deviation to mean) was used as an index of deviation from circularity. The deviation of the actual trajectory of the ankles from the horizontal plane was assessed as the percentage ratio of the maximal (the most different from zero) vertical coordinate to the relevant circle diameter.

To account for different body size and shape, the diameters were also normalised as suggested by Kerwin and Trewartha ${ }^{4}$ : all values were divided by standing height and percentage fat-free mass, and multiplied by the mean values of the group.

In addition, the angle (degrees) between the shoulder, hip, and ankle was calculated for each side of the body in each frame of movement.

\section{Statistical calculations}

Mean (SD) was calculated for each variable within the gymnast and series using either univariate or bivariate (angles) statistical analysis. Data were compared between body side (right and left), series (three repetitions), and gymnasts (five men) by analysis of variance and WatsonWilliams' test, followed by post hoc tests. Correlation coefficients were computed between anthropometric variables and diameters. Significance was set at $5 \%(p<0.05)$.

\section{RESULTS}

Within each gymnast and analysed landmark (ankle, hip, shoulder), the diameters computed in the three series were similar and symmetrical (two way factorial analysis of variance, factor 1 : series, factor 2 : body side, $\mathrm{p}>0.05$ for factors and series $\times$ side interaction). Pooled (series and side) values were computed for each gymnast. From correlation analysis, standing height explained between $84 \%$ (ankle) and $20 \%$ (hip) of the interindividual variation in the diameters, and percentage of fat-free mass explained between 65\% (hip) and $30 \%$ (ankle) of the variation.

To allow a comparison between the different gymnasts, mean diameters were normalised. All three normalised diameters were significantly different between gymnasts (table 2). In particular, gymnast M2 had the largest standardised ankle diameter and the smallest hip diameter (both values were significantly different from the other ones at post hoc analysis).

The smallest deviation from circularity of the ankle diameters was found in gymnast M2, the largest in gymnasts Ml and M4 (significant effect of gymnast, p $<0.001$, two way analysis of variance on log transformed values; no significant 
Table 2 Diameters of circular swing completed on the competition mushroom by the five gymnasts in three series of 10

\begin{tabular}{|c|c|c|c|c|c|c|c|c|c|c|c|c|c|c|}
\hline \multirow[b]{3}{*}{ Gymnast } & \multicolumn{4}{|c|}{ Series 1} & \multicolumn{4}{|c|}{ Series 2} & \multicolumn{4}{|c|}{ Series 3} & & \\
\hline & \multicolumn{2}{|l|}{$R$} & \multicolumn{2}{|l|}{$\mathbf{L}$} & \multicolumn{2}{|l|}{$\mathbf{R}$} & \multicolumn{2}{|l|}{$\mathbf{L}$} & \multicolumn{2}{|l|}{$\mathbf{R}$} & \multicolumn{2}{|l|}{ L } & \multicolumn{2}{|c|}{ Normalised $\dagger$} \\
\hline & Mean & SD & Mean & SD & Mean & SD & Mean & SD & Mean & SD & Mean & SD & Mean & SD \\
\hline Ankle & & & & & & & & & & & & & & \\
\hline MI & 1481 & 75 & 1477 & 79 & 1512 & 92 & 1517 & 88 & 1529 & 89 & 1527 & 92 & $1511^{*}$ & 86 \\
\hline M2 & 1708 & 84 & 1723 & 67 & 1729 & 65 & 1714 & 78 & 1748 & 62 & 1725 & 84 & $1671^{*}$ & 70 \\
\hline M3 & 1383 & 74 & 1378 & 64 & 1414 & 82 & 1410 & 68 & 1421 & 76 & 1413 & 68 & 1598 & 81 \\
\hline M4 & 1632 & 91 & 1610 & 105 & 1642 & 87 & 1633 & 79 & 1641 & 89 & 1630 & 89 & $1647^{*}$ & 88 \\
\hline $\begin{array}{l}\text { M5 } \\
\text { p Valuef }\end{array}$ & 1688 & 89 & 1688 & 95 & 1702 & 94 & 1701 & 92 & 1705 & 86 & 1708 & 89 & $\begin{array}{l}1535^{*} \\
0.001\end{array}$ & 79 \\
\hline MI & 285 & 87 & 268 & 84 & 288 & 93 & 280 & 88 & 294 & 92 & 281 & 97 & 283 & 87 \\
\hline M2 & 256 & 88 & 284 & 94 & 286 & 97 & 260 & 84 & 293 & 95 & 256 & 83 & $264^{*}$ & 85 \\
\hline M3 & 247 & 84 & 260 & 94 & 259 & 90 & 269 & 101 & 266 & 90 & 266 & 91 & 298 & 100 \\
\hline M4 & 299 & 105 & 320 & 109 & 302 & 107 & 312 & 106 & 306 & 101 & 310 & 114 & 311 & 104 \\
\hline $\begin{array}{l}\text { M5 } \\
\text { p Value } \\
\text { Shoulder }\end{array}$ & 344 & 101 & 327 & 93 & 332 & 92 & 348 & 106 & 347 & 102 & 333 & 102 & $\begin{array}{l}306 \\
0.042\end{array}$ & 85 \\
\hline MI & 396 & 63 & 396 & 71 & 426 & 66 & 416 & 70 & 429 & 66 & 420 & 82 & $415^{*}$ & 68 \\
\hline M2 & 520 & 92 & 509 & 60 & 513 & 73 & 528 & 91 & 512 & 62 & 524 & 85 & 454 & 132 \\
\hline$M 3$ & 362 & 63 & 358 & 74 & 380 & 79 & 379 & 93 & 382 & 60 & 379 & 61 & $425^{*}$ & 80 \\
\hline M4 & 510 & 81 & 488 & 72 & 258 & 85 & 502 & 67 & 532 & 80 & 512 & 79 & $517^{*}$ & 77 \\
\hline $\begin{array}{l}\text { M5 } \\
\text { p Value }\end{array}$ & 495 & 71 & 520 & 71 & 533 & 69 & 502 & 65 & 498 & 60 & 532 & 68 & $\begin{array}{l}459 \\
0.001\end{array}$ & 62 \\
\hline
\end{tabular}

All values are $\mathrm{mm}$

†Normalised mean diameter, mean diameter divided by height and percentage of fat-free mass, and multiplied by the mean values of the group. ¥One way analysis of variance, 2,295 degrees of freedom; ${ }^{*}$ significant difference at post hoc analysis.

Table 3 Circular swing on the competition mushroom: deviations from circularity and from the horizontal plane of the ankle trajectory

\begin{tabular}{|c|c|c|c|c|c|c|c|c|c|c|c|c|}
\hline \multirow[b]{3}{*}{ Gymnast } & \multicolumn{6}{|c|}{ Deviation from circularity } & \multicolumn{6}{|c|}{ Deviation from horizontal plane } \\
\hline & \multicolumn{2}{|c|}{ Series 1} & \multicolumn{2}{|c|}{ Series 2} & \multicolumn{2}{|c|}{ Series 3} & \multicolumn{2}{|c|}{ Series 1} & \multicolumn{2}{|c|}{ Series 2} & \multicolumn{2}{|c|}{ Series 3} \\
\hline & $R$ & $\mathbf{L}$ & $\mathbf{R}$ & L & $R$ & L & $\mathbf{R}$ & $\mathbf{L}$ & $\mathbf{R}$ & $\mathbf{L}$ & $\mathbf{R}$ & $\mathbf{L}$ \\
\hline MI & 5.0 & 5.3 & 6.1 & 5.8 & 5.8 & 6.0 & 9.6 & 13.8 & 10.5 & 14.1 & 10.8 & 18.0 \\
\hline M2 & 4.9 & 3.9 & 3.7 & 4.6 & 3.6 & 4.9 & 14.5 & 9.7 & 10.3 & 14.2 & 9.4 & 17.2 \\
\hline M3 & 5.3 & 4.6 & 5.8 & 4.8 & 5.4 & 4.8 & 14.0 & 12.6 & 19.7 & 11.8 & 14.8 & 10.1 \\
\hline M4 & 5.6 & 6.5 & 5.3 & 4.9 & 5.4 & 5.4 & 11.6 & 9.4 & 11.8 & 12.5 & 14.0 & 14.7 \\
\hline M5 & 5.3 & 5.6 & 5.5 & 5.4 & 5.1 & 5.2 & 11.1 & 13.6 & 12.9 & 14.4 & 12.8 & 10.8 \\
\hline
\end{tabular}

effect of series, no gymnast $\times$ series interaction) (table 3 ). The deviations of the same diameters from the horizontal plane were not significantly different between gymnasts or series, and no gymnast $\times$ series interaction was found.

Within gymnast and repetition, the left and right side mean angles between shoulder, hip, and ankle were symmetrical (Watson-Williams' test, $\mathrm{p}>0.05$ on all occasions), and pooled values were computed (table 4). A two way factorial analysis of variance found a significant effect of gymnast $(\mathrm{p}<0.001)$ : the largest value was found in M5 (about $155^{\circ}$ ), the smallest value in M2 (approximately $144.5^{\circ}$.

\section{DISCUSSION}

During the performance of the various techniques of gymnastics, a proper body form, including the reciprocal position of the various parts of the body in space, as well as the mode in which they move during the execution of the routine, is the key indicator of the correctness of the movements. ${ }^{124}$ This "harmony" is not usually measured, but it probably depends on the temporal and spatial characteristics of the trajectories made by the limbs, trunk, and head.

Previous quantitative investigations performed on gymnastics mainly focused on floor exercises. ${ }^{4-6}$ Data on ring

Table 4 Circular swing on the competition mushroom: angle between the shoulder, hip, and ankle

\begin{tabular}{|c|c|c|c|c|c|c|c|c|c|c|c|c|}
\hline \multirow[b]{3}{*}{ Gymnast } & \multicolumn{4}{|c|}{ Series 1} & \multicolumn{4}{|c|}{ Series 2} & \multicolumn{4}{|c|}{ Series 3} \\
\hline & \multicolumn{2}{|l|}{$R$} & \multicolumn{2}{|l|}{$\mathbf{L}$} & \multicolumn{2}{|l|}{$\mathbf{R}$} & \multicolumn{2}{|l|}{$\mathbf{R}$} & \multicolumn{2}{|l|}{$\mathrm{L}$} & \multicolumn{2}{|l|}{$\mathbf{R}$} \\
\hline & Mean & SD & Mean & SD & Mean & SD & Mean & SD & Mean & SD & Mean & SD \\
\hline M1 & 145 & 6.4 & 149 & 6.2 & 147 & 5.9 & 150 & 6.0 & 147 & 6.0 & 150 & 6.0 \\
\hline M2 & 147 & 10.7 & 143 & 10.0 & 143 & 10.8 & 146 & 11.0 & 143 & 10.2 & 145 & 11.0 \\
\hline M3 & 144 & 4.5 & 146 & 6.4 & 145 & 5.8 & 146 & 5.4 & 146 & 4.9 & 148 & 5.3 \\
\hline M4 & 147 & 7.6 & 143 & 7.2 & 148 & 6.9 & 146 & 7.8 & 151 & 6.7 & 149 & 7.2 \\
\hline M5 & 154 & 5.9 & 157 & 7.8 & 156 & 8.1 & 154 & 6.0 & 154 & 6.0 & 156 & 8.0 \\
\hline
\end{tabular}




\section{What is already known on this topic}

Three dimensional analysis of body movements in gymnastics has been used to gather values for simulation models. Previous quantitative investigations have focused mainly on floor exercises, ring routines, and the horizontal bar.

routines ${ }^{7}$ and on the horizontal bar $^{2}$ have also been reported. In contrast, the pommel horse and the competition mushroom seem to have been neglected. In this study, we focused on the ankle, hip, and shoulder movements, as well as on the three dimensional arrangement of the trunk with respect to the lower limbs during the execution of circular swings on the competition mushroom. For a correct performance of the circular swings, each ankle should follow a nearly horizontal circular path. In addition, there should be an angle of about $180^{\circ}$ at the hips, and there should be limited movement of the shoulders.

For the ankles, three measurements were performed: the diameter of the projected circle, the deviation of the trajectory from circularity, and its deviation from the horizontal plane. The dimensions of the circles depend on anthropometry (the taller the gymnast, the longer the lower limbs), muscular strength, and actual technical ability: the more the gymnast swings on the horizontal plane, the larger the projected circle. The present gymnasts had different body dimensions, and the circle diameters were normalised. ${ }^{4}$ The values reported in the last column of table 2 should therefore depend only on technical ability.

The angle between the trunk and the lower limbs was measured, as well as the diameter of the projected shoulder movement.

Overall, gymnast M2 had the largest standardised ankle diameter and the most circular trajectory. Accordingly, he had the smallest standardised hip diameter. In contrast, he had the smallest hip angle. From this point of view, the current analysis did not allow us to rank the five gymnasts analysed consistently. It should be emphasised that not all of them were currently using the competition mushroom.

The competition mushroom is used by young gymnasts (814 or 9-12 years of age depending on the country) for pommel horse training. In Italy and Germany, the apparatus is also used for local and national competitions. Unfortunately, we cannot study gymnasts younger than 18 years of age. We chose the smaller apparatus because the pommel horse was too heavy (about $90 \mathrm{~kg}$ ) to be carried into the laboratory. The use of the simpler apparatus (the competition mushroom) allowed us to extract the key values for a quantitative assessment of gymnasts performing
What this study adds

A method for analysing body movements and positions during the performance of circular swings on the competition mushroom has been developed. This may be useful to gymnasts and their coaches for determining which parts of the body are not performing the movement with sufficient accuracy and for quantifying improvements after a specific training programme.

circular swings. The same protocol could be used to appraise the actual performance on the pommel horse. At the same time, gymnasts still competing on the competition mushroom could be tested, and the method could supply useful information for the training of child and adolescent gymnasts.

The method could also help gymnasts to determine which parts of the body are not performing the movement with sufficient accuracy ${ }^{6}$ and to quantify improvements made after a specific training programme.

\section{Authors' affiliations}

G Grassi, M Turci, Y F Shirai, N Lovecchio, C Sforza, V F Ferrario,

Department of Human Morphology, University of Milan, Milan, Italy

Competing interests: none declared

\section{REFERENCES}

1 Fink H. Men's code of points. Moutier, Switzerland: Fédération Internationale de Gymnastique, 2000.

2 Takei $Y$, Nohara H, Kamimura M. Technique used by elite gymnasts in the 1992 Olympic compulsory dismount from the horizontal bar. Int J Sport Biomech 1992;8:207-32.

3 Claessens AL, Lefevre J, Beunen G, et al. The contribution of anthropometric characteristics to performance scores in elite female gymnasts. J Sports Med Phys Fitness 1999;39:355-60.

4 Kerwin DG, Trewartha G. Strategies for maintaining a handstand in the anterior-posterior direction. Med Sci Sports Exerc 2001;33:1182-8.

5 Davlin CD, Sands WA, Shults BB. Peripheral vision and back tuck somersaults. Percept Mot Skills 2001;93:465-71.

6 King MA, Yeadon MR. Coping with perturbations to a layout somersault in tumbling. J Biomech 2003;36:921-7.

7 Yeadon MR, Brewin MA. Optimised performance of the backward longswing on rings. J Biomech 2003;36:545-52.

8 Piccoli A, Brunani A, Savia G, et al. Discriminating between body fat and fluid changes in the obese adult using bioimpedance vector analysis. Int $J$ Obes 1998;22:97-104.

9 Sforza C, Turci M, Grassi G, et al. The repeatability of choku-tsuki and oi-tsuki in traditional Shotokan karate: a morphological three-dimensional analysis. Percept Mot Skills 2000;90:947-60.

10 Sforza C, Turci M, Grassi G, et al. Repeatability of mae-geri-keage in traditional karate: a three-dimensional analysis with black-belt karateka. Percept Mot Skills 2002;95:433-44. 